\title{
ANNULAR ROTATING DISKS OPTIMAL WITH RESPECT TO MIXED CREEP RUPTURE
}

\author{
ANETA UstrzycKa \\ Institute of Fundamental Technological Research, Polish Academy of Sciences, Warsaw, Poland \\ e-mail: austrzyc@ippt.pan.pl
}

KRZYSZTOF SZUWALSKI

Cracow University of Technology, Faculty of Mechanical Engineering, Institute of Applied Mechanics, Cracow, Poland

Optimal shapes in the class of polynomial functions for rotating annular disks with respect to the mixed creep rupture time are found. Two effects leading to damage: diminishing of transversal dimensions and growth of micro-cracks are simultaneously taken into account. The first of them requires the finite strain analysis, the latter is described by Kachanov's evolution equation. Behaviour of the material is described by nonlinear Norton's law, generalized for true stresses and logarithmic strains, and the shape change law in form of similarity of true stresses and logarithmic strains deviators. For optimal shapes of the disk, changes of geometry and a continuity function are presented. The theoretical considerations based on the perception of the structural components as some highlighted objects with defined properties is presented.

Keywords: annular disk, mixed creep rupture, optimal design

\section{Introduction}

The problems of optimal design in creep conditions are rather new ones, they started at the end of the last century. The difficulty, first of all, is connected with a new additional variable - time. On the other hand, such problems offer new possibilities of formulation of optimization problems Betten, 2001). Życzkowski $(1971,1988)$ pointed out some new optimization criteria such as: stress relaxation, limitation of displacements or their velocities at a given time, and time to creep rupture, which seems to be the most important one. Many solutions were found for structures with maximal time to brittle creep rupture (Białkiewicz, 1986; Ganczarski and Skrzypek, 1976; Piechnik and Chrzanowski, 1970; Rysz, 1987). Such problems are comparatively simpler, because they may be solved within the framework of the small strain theory. Optimal shapes often coincide with shapes of uniform initial strength. Much more complicated are problems of optimization using other theories of creep rupture: ductile and mixed. Both of them are connected with large deformations and require the finite strain theory. Therefore, solutions of optimal design with respect to ductile (Hoff, 1953) or mixed rupture time (Kachanov, 1960, 1999) are rather scarce. Till now, only optimal rotating bars and rotating disks with respect to ductile rupture were found by Szuwalski $(1989,1995)$. Some problems of prismatic rods under tension were discussed by Golub et al. (2008). The ductile creep rupture analysis for elastoplastic disks was carried by Dems and Mróz (1992), Ahmet and Erslan (2003), Çallığlu et al. (2006), Farshi and Bidabadi (2008), Jahed et al. (2005) and Gun (2008). Modifications of the Hoff model was proposed by Golub and Teteruk (1994). The influence of boundary conditions on the optimal shape was investigated by Pedersen (2001) and Szuwalski and Ustrzycka (2013). Even more difficult are problems of optimal design with respect to mixed creep rupture time as, besides large strains, they take into account damage development. The first solutions for optimal structures were found by Szuwalski and Ustrzycka $(2012,2013)$. 


\section{Formulation of the problem}

In the present paper, the initial shape of a rotating annular disk of given internal and external radii $A$ and $B$ and given volume $V$ (Fig. 1) ensuring the longest time to mixed creep rupture time is sought. For the annular disk of the given volume $V$, initial thickness $H$ and initial radii $A, B$ rotating with constant angular velocity $\omega$, made of a material with known properties with mass $M$ uniformly distributed at the outer edge, the optimization problem is stated as follows:

- for given $V, A, B, \omega, \gamma, M$

- we look for the initial profile of the disk $H\left(R ; b_{0}, b_{1}, b_{2}\right)=b_{0}+b_{1} R+b_{2} R^{2}$

- that $t_{*}^{(m)} \rightarrow \max$

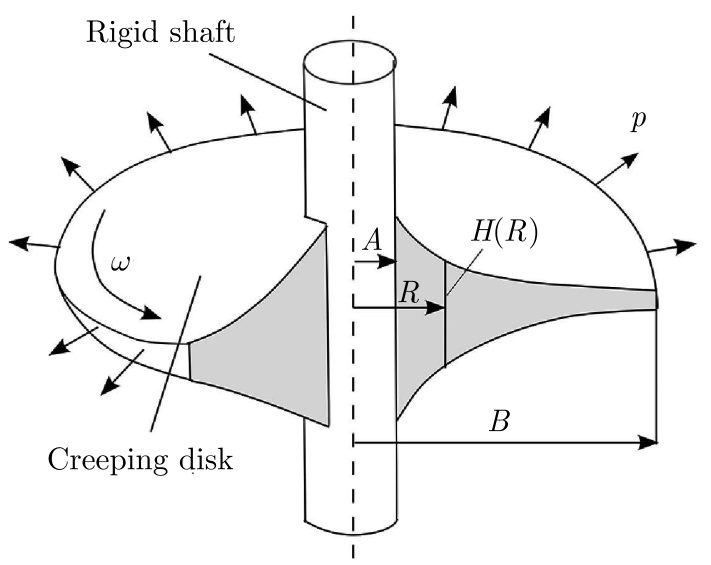

Fig. 1. Model of the annular rotating disk

The problem seems to be of great importance because in such a way rotors of jet engines, power plant turbines working in high temperatures may be calculated. In such structures, the creep effects must be taken into account, additionally of great importance are body forces. The axially symmetric problem (all variables depend only on one material coordinate, radius) is described using the material, Lagrangean coordinate denoted by capital $R$. The corresponding special coordinate $r$ is the radius of the considered point of the disk after deformation and $R$ is the radius of the considered point of the disk prior to deformation. The external loading of the disk results from the centrifugal force acting on the blades of total mass $M$ put at the outer edge of the disk on the assumption that they are uniformly distributed. Moreover, the body forces connected with own mass of the disk are taken into consideration. What makes the problem more difficult is that both loadings depend on the spatial coordinate and change within the creep process. The condition of internal equilibrium derived for an already deformed element of the disk takes form

$$
\frac{1}{h r^{\prime}} \frac{\partial}{\partial R}\left(h \sigma_{r}\right)+\frac{\sigma_{r}-\sigma_{\vartheta}}{r}+\frac{\gamma}{g} \omega^{2} r=0
$$

where $r^{\prime}$ denotes the derivative of spatial coordinate with respect to the material one, $h-$ current thickness of the disk. By "primes" in all formulas are denoted derivatives with respect to the material coordinate, while by overdots - with respect to time. The disk rotates with constant angular velocity $\omega$ and is made of a material with the specific weight $\gamma$. Radial and circumferential stresses are true stresses related to already deformed cross-sections. The stress orthogonal to the plane of the disk is disregarded (plane stress state assumption). Consequently, for large deformations, the logarithmic strains are introduced. They are connected with true stresses by Norton's law

$$
\dot{\varepsilon}_{e}=k \sigma_{e}^{n}
$$


here expressed by true stresses and logarithmic strains and generalized for complex stress state with the help of the Huber-Mises-Hencky hypothesis

$$
\sigma_{e}=\sqrt{\sigma_{r}^{2}+\sigma_{\vartheta}^{2}-\sigma_{r} \sigma_{\vartheta}}
$$

The shape change law is adopted in form of similarity of true stresses and logarithmic strains deviators, in which the creep modulus $\varphi_{c}$ is equal to

$$
\varphi_{c}=k \sigma_{e}^{n-1}
$$

This leads to the relationship

$$
\frac{\dot{r}}{r}=\frac{1}{2} k \sigma_{e}^{n-1}\left(2 \sigma_{\vartheta}-\sigma_{r}\right)
$$

Additionally, the compatibility equation for axially symmetric logarithmic strains

$$
\varepsilon_{r}=\varepsilon_{\vartheta}+\ln \left(1+R \frac{\partial \varepsilon_{\vartheta}}{\partial R}\right)
$$

expressed, with the help of the shape change law, by stresses, takes form

$$
\sigma_{\vartheta}^{\prime}=\frac{6 \sigma_{e}^{2}\left(\sigma_{r}-\sigma_{\vartheta}\right) \frac{r^{\prime}}{r}-\sigma_{r}^{\prime}\left[(n-1)\left(2 \sigma_{r}-\sigma_{\vartheta}\right)\left(2 \sigma_{\vartheta}-\sigma_{r}\right)-2 \sigma_{e}^{2}\right]}{\left.(n-1)\left(2 \sigma_{\vartheta}-\sigma_{r}\right)^{2}+4 \sigma_{e}^{2}\right]}
$$

The general assumption of incompressibility of the material may be written

$$
H R d R=h r d r
$$

Large deformations of the disk are accompanied by development of micro-cracks whose effect is described by Kachanov's evolution law

$$
\frac{\partial \Psi}{\partial t}=-D\left(\frac{\sigma_{e}}{\Psi}\right)^{m}
$$

where $\Psi$ stands for the continuity function describing the degree of damage of material, $D$ and $m$ stand for material constants. With the help of the above presented equations, the five unknowns: true radial $\sigma_{r}$ and circumferential $\sigma_{\vartheta}$ stresses, spatial coordinate $r$, current thickness $h$ and continuity function $\Psi$ may be calculated. This can be done only by numerical integration of the derived earlier equations. All unknowns are functions of two independent variables: material coordinate $R$ and time $t$. For numerical calculations, the dimensionless quantities must be introduced. The full, flat, motionless disk of volume $V$ and radius $B$ subjected to the uniform radial traction $s$ resulting from centrifugal force caused by mass $M$ uniformly distributed at radius $B$, serves as a comparative disk

$$
s=\frac{M \omega^{2}}{2 \pi h_{m}}=\frac{M \omega^{2} B^{2}}{2 V}
$$

where $h_{m}$ stands for constant thickness of the comparative disk. The dimensionless stress is related to $s(2.10)$, whereas the dimensionless time is defined as

$$
\widehat{t}=\frac{t}{t_{0}^{(d)}}
$$

where $t_{0}^{(d)}$ stands for the time of ductile rupture for the full plane disk. To evaluate the time of ductile rupture $t_{0}^{(d)}$ for the full plane disk, the following equation may be used

$$
\dot{\varepsilon}_{z}=\frac{\dot{h}}{h}=\frac{3}{2} k \sigma_{e}^{n-1}\left(\sigma_{z}-\sigma_{m}\right)
$$


In the problem under consideration, the effective stress is

$$
\sigma_{e}=\sigma_{r}=\sigma_{\vartheta}=p
$$

while the mean stress

$$
\sigma_{m}=\frac{2}{3} p
$$

The implementation of these substitutions to (2.12) leads to the equation

$$
\frac{1}{h} \frac{d h}{d t}=-k\left(\frac{M \omega^{2}}{2 \pi h}\right)^{n}
$$

describing the change of thickness in time. The initial condition takes form

$$
\text { for } \quad t=0 \quad h=h_{m}
$$

The condition of ductile rupture: $h \rightarrow 0$, enables calculation of the time of ductile rupture $t_{0}^{(d)}$

$$
t_{0}^{(d)}=\frac{1}{n k\left(\frac{M \omega^{2}}{2 \pi h_{m}}\right)^{n}}=\frac{1}{n k s^{n}}
$$

Finally, dimensionless time (2.11) is defined as

$$
\widehat{t}=n k s^{n} t
$$

The dimensionless radii are related to external radius $B$ of the comparative disk and dimensionless thickness to its constant thickness $h_{m}$.

Finally, the set of five differential dimensionless equations takes form

$$
\begin{aligned}
\widehat{\sigma}_{r}^{\prime} & =\frac{\widehat{r}^{\prime}}{\widehat{r}}\left(\widehat{\sigma}_{\vartheta}-\widehat{\sigma}_{r}\right)-2 \widehat{r}^{\prime} \mu-\frac{\widehat{h}^{\prime}}{\widehat{h}} \widehat{\sigma}_{r} \\
\widehat{\sigma}_{\vartheta}^{\prime} & =\frac{6 \widehat{\sigma}_{e}^{2}\left(\widehat{\sigma}_{r}-\widehat{\sigma}_{\vartheta}\right) \frac{\widehat{r}^{\prime}}{\widehat{r}}-\widehat{\sigma}_{r}^{\prime}\left[(n-1)\left(5 \widehat{\sigma}_{r} \widehat{\sigma}_{\vartheta}-2 \widehat{\sigma}_{r}^{2}-2 \widehat{\sigma}_{\vartheta}^{2}\right)-2 \widehat{\sigma}_{e}^{2}\right]}{(n-1)\left(2 \widehat{\sigma}_{\vartheta}-\widehat{\sigma}_{r}\right)^{2}+4 \widehat{\sigma}_{e}^{2}}
\end{aligned}
$$

and

$$
\frac{d \widehat{r}}{d \widehat{t}}=\frac{\widehat{r}}{2 n}\left(\widehat{\sigma}_{r}^{2}+\widehat{\sigma}_{\vartheta}^{2}-\widehat{\sigma}_{r} \widehat{\sigma}_{\vartheta}\right)^{\frac{n-1}{2}}\left(2 \widehat{\sigma}_{\vartheta}-\widehat{\sigma}_{r}\right) \quad \widehat{h}=\frac{\widehat{H} \widehat{R}}{\widehat{r}^{\prime} \widehat{r}}
$$

and

$$
\frac{\partial \Psi}{\partial \widehat{t}}=\frac{-1}{(m+1) \Theta}\left(\frac{\widehat{\sigma}_{e}}{\Psi}\right)^{m}
$$

where all dimensionless quantities are denoted by $(\widehat{\bullet})$ over the symbol, and additional parameters $\mu$ and $\Theta$ are introduced

$$
\mu=\frac{\gamma V}{g M}
$$

where $\mu$ is the ratio of own mass of the disk to the mass $M$ uniformly distributed at the outer edge, and $\Theta$ contains and replaces four material constants from Norton's law and the evolution equation

$$
\Theta=\frac{t_{p r}^{(b)}}{t_{p r}^{(d)}}=\frac{n k}{(m+1) D} s^{n-m}
$$


and is defined as the ratio of brittle and ductile creep rupture time of prismatic bars subject to tension with stress $s(2.10)$. This parameter may be also treated as a measure of sensitivity on the kind of creep rupture.

We define the rupture criterion in the following form

$$
\exists R:\left.\quad R \in\langle 0, l\rangle \wedge \Psi \in\langle 1,0\rangle \wedge \Psi(\sigma)\right|_{t_{*}^{(m)}} \rightarrow 0
$$

The time after which the continuity function diminishes to zero is the time of mixed rupture $t_{*}^{(m)}$. The criterion of rupture is adopted in form of diminishing to zero the value of the continuity function at least at one nodal point.

\section{Numerical calculations}

To obtain unique solutions for the above presented set of differential equations, initial and boundary conditions must be formulated. At the beginning of the creep process, the shape of the disk is described by the assumed equation $H(R)$. As the initial condition serves the coincidence of the spatial and material coordinate

$$
\widehat{r}(\widehat{R}, 0)=\widehat{R} \quad \widehat{h}(\widehat{R}, 0)=\widehat{H}(\widehat{R})
$$

The boundary condition at the inner radius $A$ may be formulated in several ways. In the case when the disk is connected with a rigid shaft

$$
\widehat{\sigma}_{r}(\beta, \widehat{t})=2 \widehat{\sigma}_{\vartheta}(\beta, \widehat{t})
$$

the radial stress is two times larger than the circumferential one, but their values are unknown. The parameter $\beta$ stands for the ratio of radii of the disk: $\beta=A / B$. On the other hand, at the outer radius, the radial stress must be equal to tension caused by the centrifugal force acting on the mass $M$ placed there

$$
\widehat{\sigma}_{r}(1, \widehat{t})=\frac{1}{\widehat{h}(1, \widehat{t})}
$$

For this reason, a recurrential procedure must be used. Assuming various values of stresses at the radius $A$, we must finally find such values which will make it possible to satisfy condition (3.3). The numerical algorithm consists of three steps:

- first step - for given geometry of the disk, the true stresses distribution is established (from Eqs. (2.19)). We do not know the values of stresses at the inner edge of the disk, so we assume them arbitrarily. The boundary condition at the outer edge of the disk must be fulfilled (see Fig. 2).

- second step - from the first step, the distribution of true stresses is known. It is possible to establish the new, changed geometry of the disk (from Eqs. (2.20)). The mixed creep rupture can occur earlier than the ductile creep rupture. Therefore, it is necessary to check whether the thickness is not small enough $\left(h_{a d m}\right)$, which finishes the procedure.

- third step - the distribution of the continuity function $\Psi$ is calculated from Eq. (2.21). If its minimum value satisfies the rupture criterion, the creep process is finished - the time to mixed rupture has been found. 


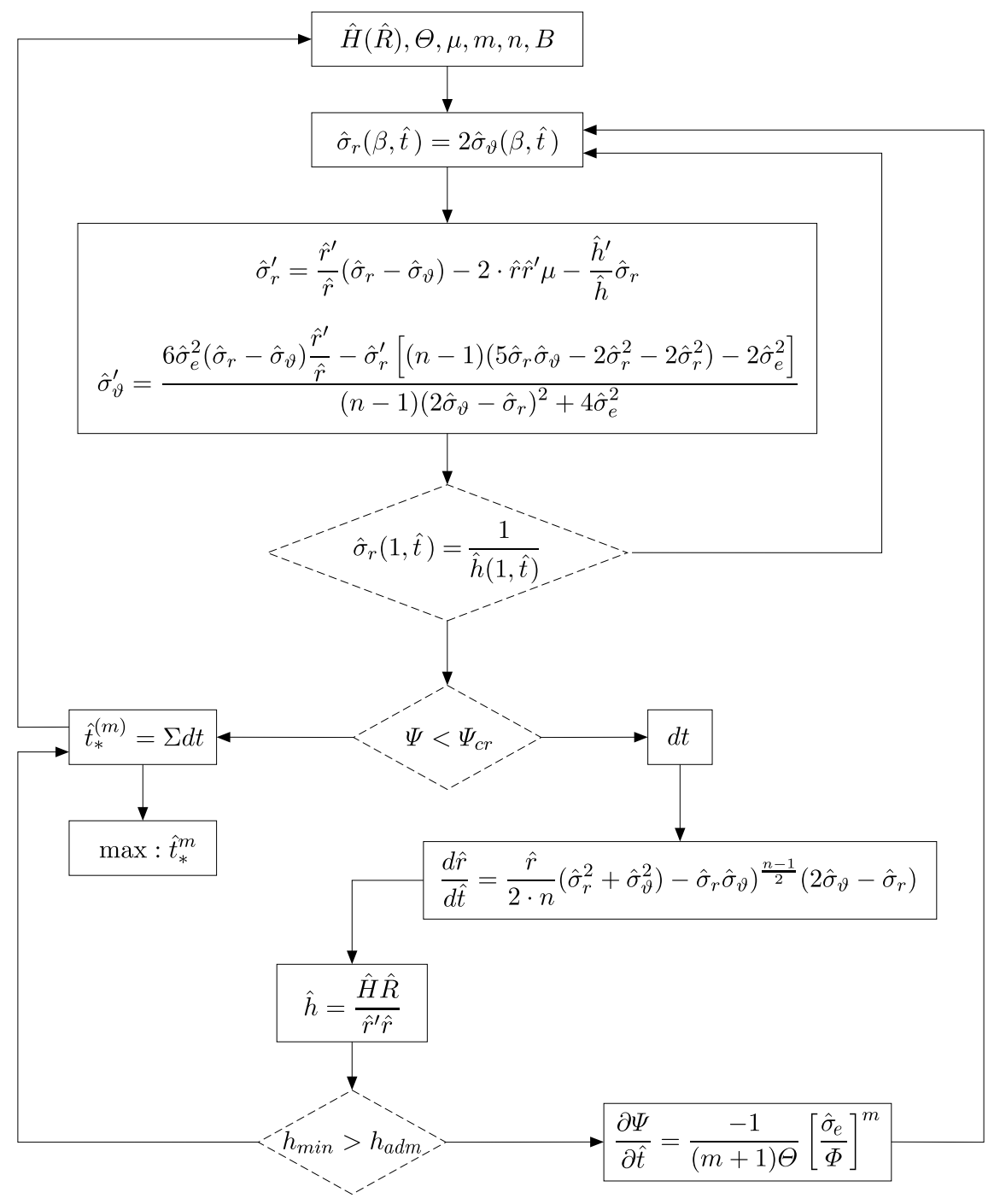

Fig. 2. Algorithm of numerical calculations - flow chart

\section{Uniparametric optimization}

Due to the inability of explicitly writting the objective function (mixed rupture time) as a function of the optimization parameters (initial profile of the disk), the parametric optimization is applied (search method). Initially, the optimal solution is sought among conical disks whose initial shape is described by the formula

$$
\widehat{H}\left(\widehat{R} ; u_{0}, u_{1}\right)=u_{0}+u_{1} \widehat{R}
$$

From the condition of constant volume this leads to

$$
u_{1}=\frac{3}{2}\left(1-u_{0}\right)
$$

so only one free parameter $u_{0}$ remains.

\subsection{Conical disk clamped on a rigid shaft}

The optimal solutions of the disks clamped on a rigid shaft for various parameters $\Theta$ are presented in Fig. 3. 


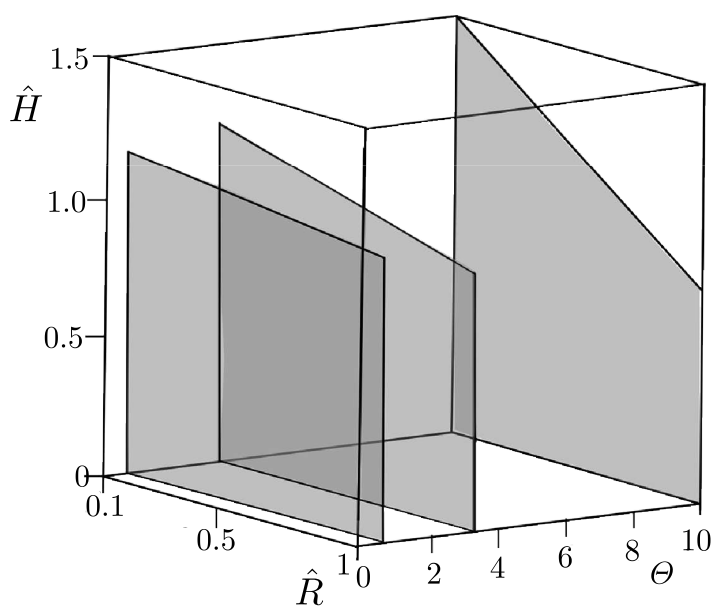

Fig. 3. Optimal profiles of a conical disk clamped on a rigid shaft, $\beta=0.1, \mu=0.1$

For $\Theta=0.4$ the optimal profile of the conical disk becomes almost flat. For higher values of the parameter $\Theta$, the mass moves toward the inner edge.

\subsection{Conical disk with the free inner edge}

The boundary condition at the inner radius is described in the following way

$$
\widehat{\sigma}_{r}(\beta, \widehat{t})=0
$$

and at the outer radius by the following equations

$$
\widehat{\sigma}_{r}(1, \widehat{t})=\frac{1}{\widehat{h}(1, \widehat{t})}
$$

The optimal profiles for the conical disk found according to the algorithm presented in Fig. 2 as a function of the parameter $\Theta$ are plotted in Fig. 4 .

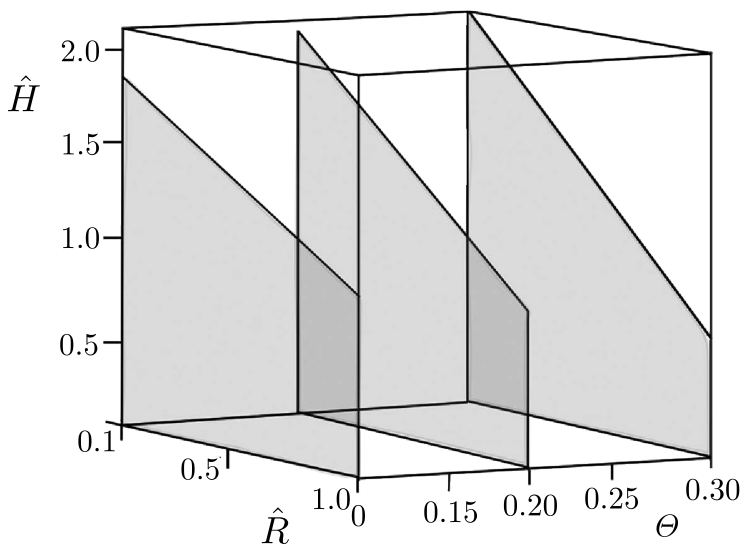

Fig. 4. Optimal profiles of the conical disk with the free inner edge, $\beta=0.1, \mu=0.1$

The optimal profiles of conical disks with the free inner edge are characterized by a significant reduction of thickness at the outer radius, even larger than that for the disk clamped on a rigid shaft. 


\section{Biparametric optimization}

It is expected that the earlier presented results could be improved by expanding the class of functions, in which the optimal solution is sought. In the next step, biparametric optimization is used. The initial shape is defined by a quadratic function

$$
\widehat{H}\left(\widehat{R} ; b_{0}, b_{1}, b_{2}\right)=b_{0}+b_{1} \widehat{R}+b_{2} \widehat{R^{2}} \quad b_{2} \neq 0
$$

From the three parameters in this function, only two may be treated as free ones, the third results from the given volume of the disk

$$
b_{2}=2-2 b_{0}-\frac{4}{3} b_{1}
$$

The search process for biparametric optimization is much more time-absorbing. For the given parameter $b_{0}$, the time to the mixed rupture for various parameters $b_{1}$ is calculated. In such a way one may find the parameter $b_{1}$ leading to the longest lifetime. This procedure is repeated for subsequent values $b_{0}$. At last the optimal solution is established as "maximum maximorum" of all investigated disks (sometimes almost hundred).

\subsection{Parabolic disk clamped on a rigid shaft}

The creep process of the annular rotating disk is considered for three different boundary conditions. As the first, the disk clamped on a rigid shaft will be analyzed (e.g. welded connections). Then, despite condition (3.2), an additional condition must be satisfied

$$
\widehat{h}(\beta, \widehat{t})=\widehat{H}(\beta, 0)
$$

Optimal shapes of the disks for biparametric optimization are shown in Fig. 5.

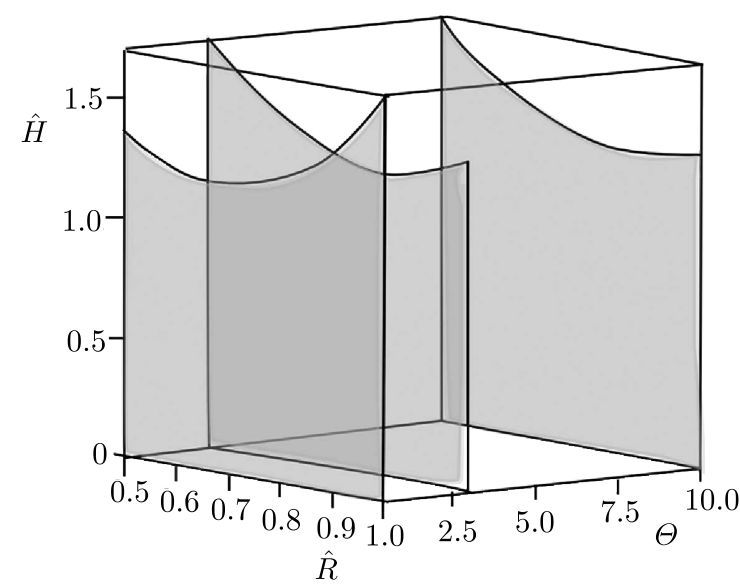

Fig. 5. Optimal shapes of disks clamped on the rigid shaft, $\beta=0.5, \mu=0.1$

For $\Theta=0.4$ the optimal shape of the disk is characterized by a large increase of thickness at the external edge. In spite of larger centrifugal forces, the outer edge works as a kind of reinforcement slowing down the creep process. For larger values of the parameter $\Theta$, this effect does not occur.

The course of the creep process for finding the optimal disk with the initial profile described by the function

$$
\widehat{H}(\widehat{R})=3.51-5 \widehat{R}+2.76 \widehat{R}^{2}
$$


is presented in Fig. 6 showing changes of the shape (a) and of the distribution of the continuity function (b) in terms of time. The distribution of the continuity function at the moment of rupture is not uniform, the criterion of rupture is fulfilled inside the disk at one point. For other radii, values of the function $\Psi$ are non-zero, and at the inner and outer edge they are quite large. This effect is related only to disks with the initial profile described by quadratic function (5.4).

(a)

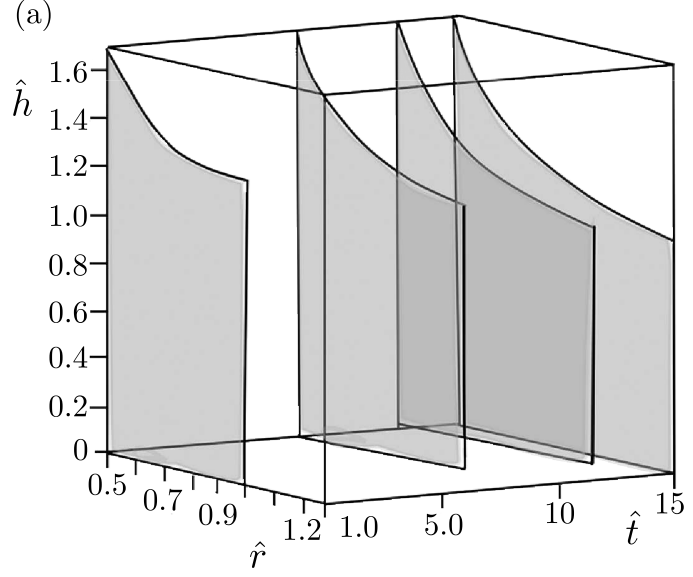

(b)

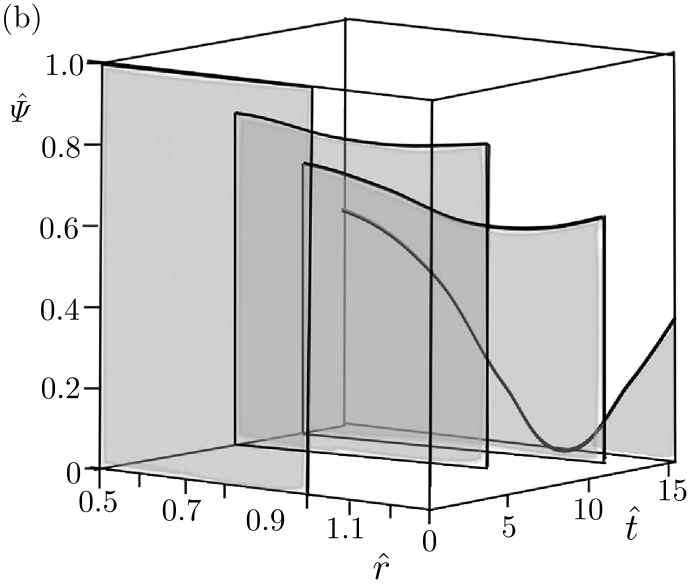

Fig. 6. Time cross-section of the creep process for $\beta=0.5, \mu=0.1$ and $\Theta=3$

\subsection{Parabolic disk fastened on the rigid shaft with thickness allowed to change}

Optimal shapes of disks fastened on a rigid shaft (radial displacement is possible) but with possibility of thickness changing (e.g. spline joint) are shown in Fig. 7.

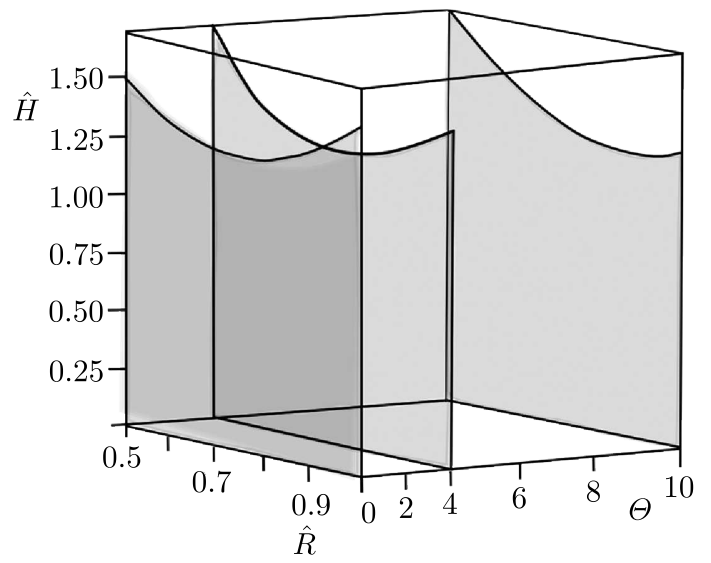

Fig. 7. Optimal shapes of disks fastened on the rigid shaft $\beta=0.5, \mu=0.1$

For $\Theta=0.4$ and $\Theta=3$ the optimal shapes of the disk have a reinforcement of the outer edge (increase of the thickness). For $\Theta=10$ this effect is smaller.

The above figure drawn for the optimal disk

$$
\widehat{H}(\widehat{R})=4.39-8 \widehat{R}+5.05 \widehat{R}^{2}
$$

shows the time cross-section of the optimal profile of the annular disk fastened on the rigid shaft (Fig. 8a) and the continuity function (Fig. 8b). The geometrical changes of the profile of the disk in the creep process are not significant. Due to small width of the disk, the change of thickness at the inner edge, although permissible, is not too large. 
(a)

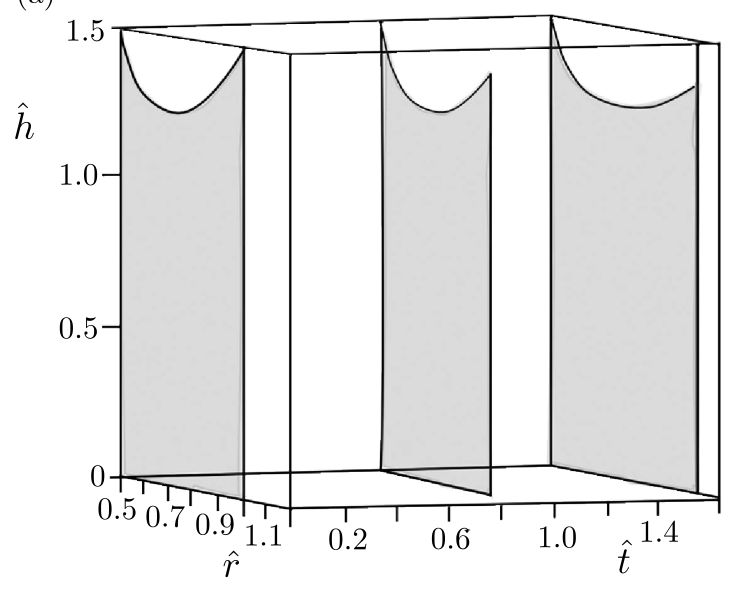

(b)

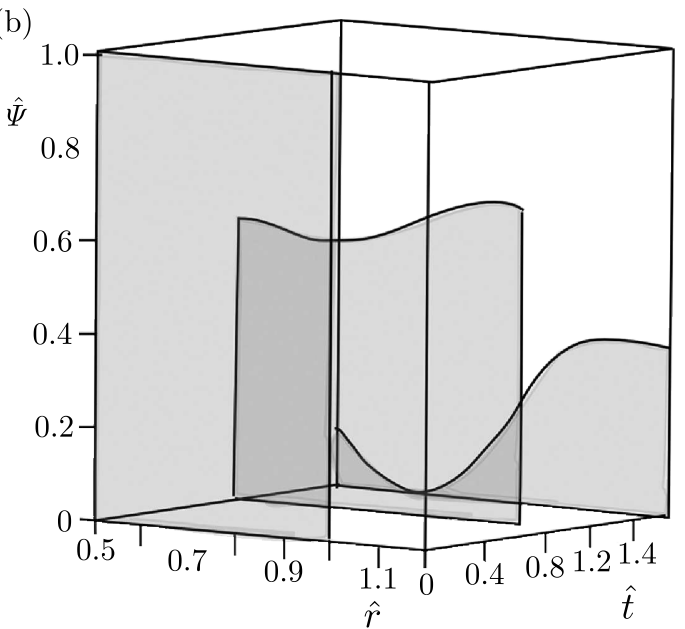

Fig. 8. Time cross-section of the creep process for $\beta=0.5, \mu=0.1, \Theta=3$

\subsection{Parabolic disk with the free inner edge}

Optimal shapes of the free disks (4.3) for biparametric optimization are shown in Fig. 9.

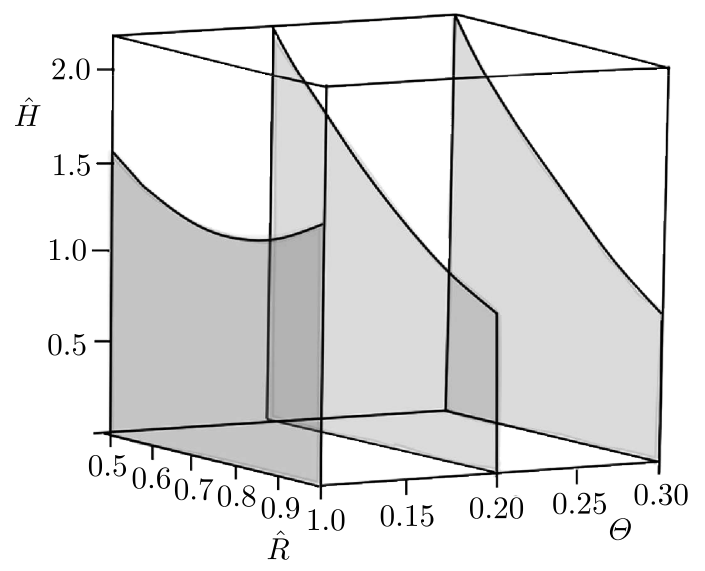

Fig. 9. Optimal shape of the disk with the free inner edge, $\beta=0.5, \mu=0.1$

The reinforcement of the outer edge of the disk is observed for $\Theta=0.1$. For $\Theta=0.2$ and $\Theta=0.3$ the optimal shapes are characterized by reduction of the thickness of the disk toward the outer edge.

As an example of the found optimal profiles is

$$
\widehat{H}(\widehat{R})=4.09-5.01 \widehat{R}+1.79 \widehat{R}^{2}
$$

The time cross-section for this optimal profile of the free disk and the continuity function is shown respectively in Figs. 10a and 10b.

The time cross sections of the continuity function indicate that the criterion of rupture of the disk is fulfilled at the inner edge, despite the significant reinforcement of thickness at this point.

\section{Corrected disks of uniform initial strength}

We expect that disks of uniform initial strength, in which the radial and circumferential stresses are equal and independent of radius, would be close to the optimal profiles with respect to the 

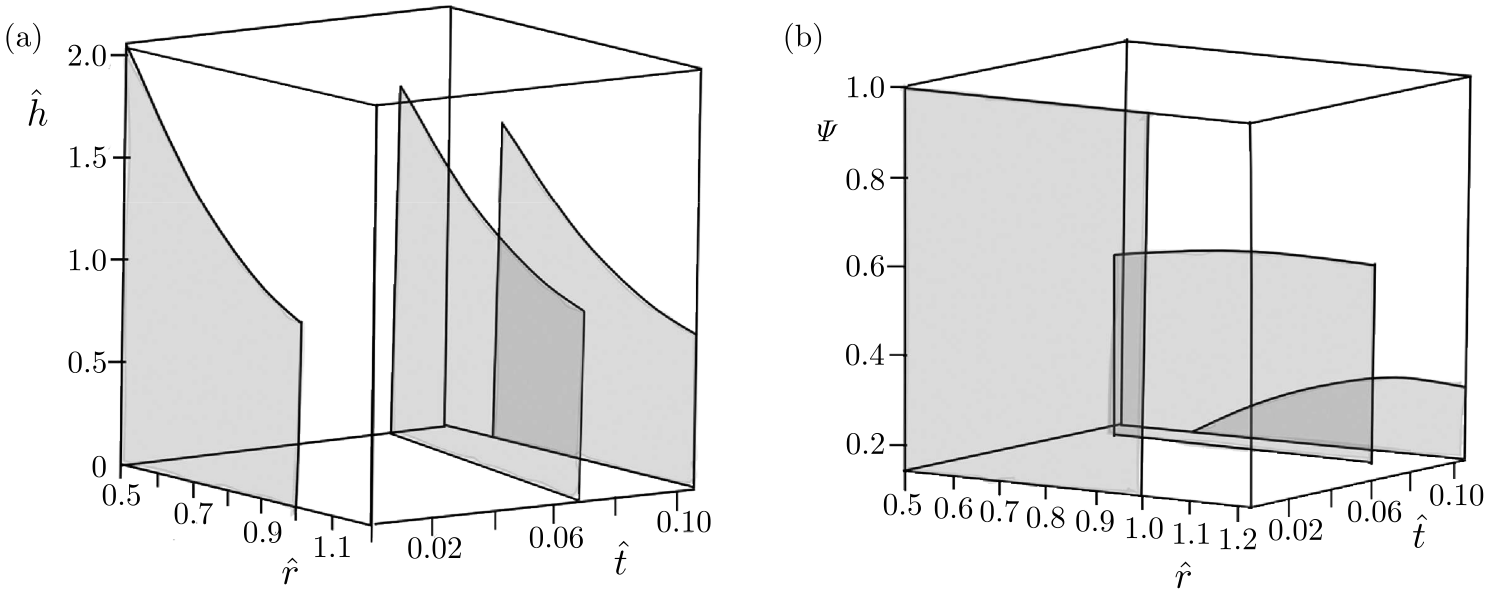

Fig. 10. Time cross-sections of the creep process for the free edge, $\Theta=0.2, \beta=0.5, \mu=0.1$

mixed rupture time. For a homogeneous material of the uniform initial strength disk, for $t=0$, the radial $\Sigma_{r}$ and circumferential $\Sigma_{\vartheta}$ stresses are the same

$$
\Sigma_{r}=\Sigma_{\vartheta}=\Sigma=\operatorname{const}(R)
$$

and the stress perpendicular to the middle surface of the disk is assumed to be equal to zero $\left(\sigma_{z} \equiv 0\right)$.

The shape of uniform initial strength disks results from the internal equilibrium equation

$$
\widehat{H}_{u s}(\widehat{R})=\frac{1}{\widehat{\Sigma}} \exp \left[\frac{\mu}{\widehat{\Sigma}}\left(1-\widehat{R}^{2}\right)\right]
$$

where $\widehat{\Sigma}$ denotes the dimensionless equalized initial stress calculated from the condition of constant volume

$$
\widehat{\Sigma}=\frac{\mu}{\ln (1+\mu)}
$$

The correction of shape (6.2) is imposed, which is adopted here in form of a third degree polynomial function without linear term

$$
\widehat{H}_{\text {cor }}=p_{0}+p_{2} \widehat{R}^{2}+p_{3} \widehat{R}^{3}
$$

Finally, the initial profile of the disk is described by the equation

$$
\widehat{H}(\widehat{R})=\widehat{H}_{u s}(\widehat{R})+\widehat{H}_{\text {cor }}(\widehat{R})
$$

Numerical calculations have been carried out for the set of parameters: $\mu=0.1, \Theta=3$, and exponents: in Norton's law $n=6$, in Kachanov's law $m=2$. It turns out that the optimal correction takes form

$$
\widehat{H}_{\text {cor }}=-0.3-0.04 \widehat{R}^{2}+9.47 \widehat{R}^{3}
$$

The optimal shape of the corrected disk of uniform initial strength compared with the profiles obtained with uni- and biparametric optimizations is presented in Fig. 11. Additionally, the time to rupture for all those solutions is given.

The corrected profile of uniform initial strength disks provides the longest time to mixed rupture. It is characterized by significant strengthening of the middle and outer part of the disk, which seems to be very reasonable taking into account the results shown in Fig. 11. 


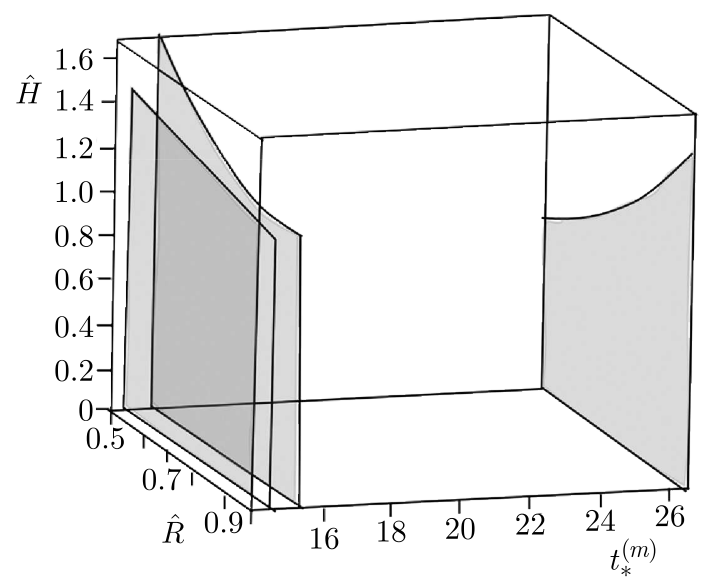

Fig. 11. Optimal shape of the uniform initial strength disks compared with uni- and biparametric optimization

\section{Conclusions}

The problems of optimal design in creep conditions are very difficult due to a new additional factor-time. When the time to ductile or mixed creep rupture is involved, the difficulties even grow. Such problems require the finite strain approach, i.e. resignation of the rigidification theorem and analysis of the already deformed body using true stresses and logarithmic strains. For this reason, the parametric optimization is applied - the initial shape of the disk is described by a polynomial function. The obtained solutions strongly depend on boundary conditions at the inner edge of the annular disk. For disks fastened on a rigid shaft, the time to rupture is much longer than for disks with free inner edges. Better results - longer times to mixed creep rupture are obtained for biparametric optimization - the initial shape described by a quadratic function. The best results are obtained for the disk of uniform initial strength corrected by a polynomial function of the third degree. A significant influence on the optimal solution of the parameter $\Theta$ describing the sensitivity of the material to brittle or ductile rupture is observed. Also the ratio of own mass of the disk and mass placed at the outer edge $\mu$ is of great importance.

\section{Acknowledgement}

This work has been supported by the National Science Centre through the Grant No. 2014/15/B/ST8/04368.

\section{References}

1. Ahmet N., Eraslan., 2003, Elastic-plastic deformations of rotating variable thickness annular disks with free, pressurized and radially constrained boundary conditions, International Journal of Mechanical Sciences, 45, 4, 643-667

2. Betten J., 2001, Mathematical modeling of materials behavior under creep conditions, Applied Mechanics Reviews, 54, 2, 107-132

3. BiAŁkiEWiCZ J., 1986, Dynamic creep rupture of a rotating disk of variable thickness, International Journal of Mechanical Sciences, 28, 10, 671-681

4. ÇAllioĞLu H., Topcu M., TARAKCilar A.R., 2006, Elastic-plastic stress analysis on orthotropic rotating disc, International Journal of Mechanical Sciences, 48, 985-990

5. Dems K., Mróz Z., 1992, Shape sensitivity analysis and optimal design of disks and plates with strong discontinuities of kinematic fields, International Journal of Solids and Structures, 29, 4, $437-463$ 
6. FArshi B., Bidabadi J., 2008, Optimum design of inhomogeneous rotating discs under secondary creep, International Journal of Pressure Vessels and Piping, 85, 507-515

7. Ganczarski A., Skrzypek J., 1976, Optimal shape of prestressed disks in creep, Journal of Structural Mechanics, 2, 141-160

8. Golub V.P., Romanov A.V., Romanova N.V., 2008, Nonlinear creep and ductile creep rupture of perfectly elastoplastic rods under tension, International Applied Mechanics, 44, 4, 459-470

9. Golub V.P., Teteruk R.G., 1994, Evaluating the time to ductile fracture under creep conditions, International Applied Mechanics, 30, 11, 898-905

10. Gun H., 2008, Two-dimensional boundary element analysis of creep continuum damage problems with plastic effects, Computational Materials Science, 41, 3, 322-329

11. Hoff N.J., 1953, The necking and rupture of rods subjected to constant tensile loads, Journal of Applied Mechanics - Transactions of ASME, 20, 105-112

12. Jahed H., Farshi B., Bidabadi J., 2005, Minimum weight design of inhomogeneous rotating discs, International Journal of Pressure Vessels and Piping, 82, 35-41

13. Kachanov L.M., 1960, Creep Theory, Fizmatgiz, Moskow

14. Kachanov L.M., 1999, Rupture time under creep conditions, International Journal of Fracture, 97, xi-xviii

15. Pedersen P., 2001, On the influence of boundary conditions, Poisson's ratio and material nonlinearity on the optimal shape, International Journal of Solids and Structures, 38, 3, 465-477

16. Piechnik S., Chrzanowski M., 1970, Time of total creep rupture of a beam under combined tension and bending, International Journal of Solids and Structures, 6, 4, 453-477

17. Rysz M., 1987, Optimal design of a thick-walled pipeline cross-section against creep rupture, Acta Mechanica, 1, 4, 83-102

18. Szuwalski K., 1989, Optimal design of bars under nonuniform tension with respect to ductile creep rupture, Mechanics of Structures and Machines, 3, 303-319

19. SzuWAlski K., 1995, Optimal design of disks with respect to ductile creep rupture time, Structural Optimization, 10, 54-60

20. Szuwalski K., UstrzyckA A., 2012, Optimal design of bars under nonuniform tension with respect to mixed creep rupture time, International Journal of Non-Linear Mechanics, 47, 55-60

21. Szuwalski K., Ustrzycka A., 2013, The influence of boundary conditions on optimal shape of annular disk with respect to ductile creep rupture time, European Journal of Mechanics, 37, 79-85

22. ŻyCZkowski M., 1971, Optimal structural design in rheology, Journal of Applied Mechanics, 38, $1,39-46$

23. Życzkowski M., 1988, Optimal structural design under creep conditions, Applied Mechanics Reviews, 12, 453-461 